\title{
One-loop quantum gravity from a worldline viewpoint
}

\author{
Fiorenzo Bastianelli and Roberto Bonezzi \\ Dipartimento di Fisica e Astronomia, Università di Bologna and \\ INFN, Sezione di Bologna, via Irnerio 46, I-40126 Bologna, Italy \\ E-mail: bastianelli@bo.infn.it, bonezzi@bo.infn.it
}

\begin{abstract}
:
We develop a worldline approach to quantum gravity in $D=4$. Using the background field method we consider the covariantly gauge fixed Einstein-Hilbert action with cosmological constant, and find a worldline representation of the differential operators identified by its quadratic approximation. We test it by computing the correct one-loop divergencies. Alternative worldline methods, such as the use of the $O(4)$ spinning particle that is known to describe correctly the propagation of a massless spin 2 particle in $D=4$, find obstructions in the coupling to an arbitrary background metric, apparently preventing a more extensive use in perturbative descriptions of quantum gravity. We expect that our model might simplify calculations of one-loop amplitudes with respect to standard quantum field theoretical methods.
\end{abstract}

KeYwords: Models of Quantum Gravity, Sigma models, Gauge Symmetry. 


\section{Contents}

1 Introduction 1

2 Background-quantum splitting of Einstein-Hilbert action 2

3 Gauge fixing 4

4 Effective action $\quad 5$

$\begin{array}{lll}5 & \text { Worldline representation } & 6\end{array}$

$\begin{array}{ll}5.1 \text { The traceless tensor model } & 7\end{array}$

$\begin{array}{ll}5.2 \text { The vector model } & 10\end{array}$

6 One-loop effective action from worldline path integrals $\quad 12$

6.1 Traceless tensor path integral 13

$\begin{array}{ll}6.2 \text { Vector path integral } & 16\end{array}$

$\begin{array}{lll}6.3 & \text { Final result } & 17\end{array}$

$\begin{array}{lr}\text { A Fermionic coherent states } & 18\end{array}$

$\begin{array}{lr}\text { B Dimensional regularization } & 19\end{array}$

\section{Introduction}

Worldline methods have been useful for studying quantum field theoretical processes both in flat space (see [1] for a review) and in curved space (see [2-6] for examples). However, the inclusion of a quantum spin 2 particle to account for the graviton has proved difficult to achieve. The use of the $\mathrm{O}(4)$-extended spinning particle ${ }^{1}$, which is certainly able to describe the propagation of a massless spin 2 particle in flat space, has met with severe obstructions in trying to couple it to a generic curved background. Indeed in [9] and [10] it was found how to couple it to (A)dS and conformally flat spaces, respectively, but an extension to generic background metrics remains elusive.

This problem is in a way similar to the case of the $\mathrm{O}(2)$-extended spinning particle, that can be used to describe spin 1 fields coupled to background gravity, but which does not seem to admit a coupling to background gauge fields. Nevertheless, it has been used successfully in [11] to give a worldline description of non-abelian fields by using BRST

\footnotetext{
${ }^{1}$ It belongs to the class of $\mathrm{O}(N)$-extended spinning particles that describe particles of spin $N / 2[7,8]$. For $N=0,1,2$ they have been used to describe spin $0,1 / 2$, and 1 coupled to curved backgrounds in refs. $[2],[3]$, and $[4,5]$, respectively.
} 
and string inspired methods. The extension of these methods to the $\mathrm{O}(4)$ particle is not obvious.

Given this state of affairs, we try here a simpler, though perhaps less elegant, approach, namely that of deriving from the quadratic fluctuations of the gravitational field suitable particle actions that upon quantization give precisely the differential operators of the quadratic fluctuations. We keep an arbitrary background metric and adopt a convenient background-covariant gauge fixing. This approach has the virtue of allowing for a useful representation of the one-loop effective action of quantum gravity in terms of worldline path integrals. We test this method by reproducing the correct one-loop divergencies for the Einstein gravity [12], in the presence of the cosmological constant as well [13] (see also [14]).

These standard results can be used to test the correctness of the worldline calculation of the heat kernel coefficients in the one-loop effective action of higher spin fields on (A)dS spaces, recently performed in [15] by use of the $\mathrm{O}(N)$-extended spinning particles. One may check that in the case of spin 2 in $D=4$ the coefficient of the logarithmic singularity found in [15] differs from the one reported in [13]. However that was not totally unexpected, as one typically finds topological mismatches for dually related theories in such a coefficient, which is indeed proportional to the Euler character in $D=4$, see $[4,16]$ for the case of differential forms. The quadratic (and quartic) divergences on the other hand are not topological, but were not reported in [13, 14], so that a direct test could not be performed in [15]. We find now that they indeed agree with the ones explicitly recomputed with our new method in the present paper.

We expect that the flexibility of the worldline path integral representation might be useful in calculating one-loop quantum gravity processes in a simpler way than standard QFT methods. Of course, there are many powerful approaches specific to $D=4$ that are being considered in the literature nowadays. The present work is a step towards the understanding of the role the worldline formalism can play in describing quantized gravitons. Yet, it would be interesting to find a unified worldline model able to describe the graviton on its own, and not coming directly from manipulating QFT expressions. The $\mathrm{O}(4)$ spinning particle indeed describes spin 2 massless particles, but it does not allow them to interact with an arbitrary gravitational background, and something new is perhaps needed in that direction.

\section{Background-quantum splitting of Einstein-Hilbert action}

Let us consider a $D$ dimensional curved manifold $\mathcal{M}$ with euclidean signature, equipped with a riemannian metric $G_{\mu \nu}(x)$. The Einstein-Hilbert action with cosmological constant reads

$$
S[G]=-\frac{1}{\kappa^{2}} \int d^{D} x \sqrt{G}[R(G)-2 \Lambda] .
$$

We perform a background-quantum splitting by defining a fixed classical metric $g_{\mu \nu}(x)$ and quantum metric fluctuations $h_{\mu \nu}(x)$, such that $G_{\mu \nu}(x)=g_{\mu \nu}(x)+h_{\mu \nu}(x)$. In order to get the effective action at one-loop, it is sufficient to consider the perturbative expansion of 
(2.1) up to quadratic order in $h_{\mu \nu}$. Expanding the Einstein-Hilbert action in powers of $h_{\mu \nu}$ we get

$$
S[g+h]=\frac{1}{\kappa^{2}}\left[S_{0}+S_{1}+S_{2}+\sum_{n=3}^{\infty} S_{n}\right],
$$

where

$$
\begin{aligned}
S_{0} & =-\int d^{D} x \sqrt{g}\{R-2 \Lambda\} \\
S_{1} & =\int d^{D} x \sqrt{g}\left\{h^{\mu \nu}\left(R_{\mu \nu}-\frac{1}{2} g_{\mu \nu} R+g_{\mu \nu} \Lambda\right)\right\} \\
S_{2} & =-\int d^{D} x \sqrt{g}\left\{\frac{1}{4} h^{\mu \nu}\left(\nabla^{2}+2 \Lambda\right) h_{\mu \nu}-\frac{1}{8} h\left(\nabla^{2}+2 \Lambda\right) h+\frac{1}{2}\left(\nabla^{\nu} h_{\nu \mu}-\frac{1}{2} \nabla_{\mu} h\right)^{2}\right. \\
& \left.+\frac{1}{2} h^{\mu \lambda} h^{\nu \sigma} R_{\mu \nu \lambda \sigma}+\frac{1}{2}\left(h^{\mu \lambda} h_{\lambda}^{\nu}-h h^{\mu \nu}\right) R_{\mu \nu}+\frac{1}{8}\left(h^{2}-2 h^{\mu \nu} h_{\mu \nu}\right) R\right\}
\end{aligned}
$$

with $h \equiv g^{\mu \nu} h_{\mu \nu}$. In the above formulas and from now on all covariant derivatives and curvature tensors are intended to be background quantities built from $g_{\mu \nu}$ only: $\nabla \equiv \nabla(g)$ and so on. The indices are raised and lowered by means of $g_{\mu \nu}$ and its inverse, so that $h_{\nu}^{\mu} \equiv g^{\mu \sigma} h_{\sigma \nu}$ and so forth.

The original action (2.1) of course enjoys invariance under diffeomorphisms of the full metric

$$
\delta G_{\mu \nu}=£_{\xi} G_{\mu \nu}=\xi^{\lambda} \partial_{\lambda} G_{\mu \nu}+\left(\partial_{\mu} \xi^{\lambda}\right) G_{\lambda \nu}+\left(\partial_{\nu} \xi^{\lambda}\right) G_{\mu \lambda}
$$

where $£_{\xi}$ denotes the Lie derivative along the vector field $\xi^{\mu}$. In the split action (2.2) one can recognize two different symmetries, both taking the same form (2.4) when acting on the full $G_{\mu \nu}$ : a classical symmetry under background diffeomorphisms of $g_{\mu \nu}$, with $h_{\mu \nu}$ transforming as a tensor, i.e.

$$
\delta_{\xi} g_{\mu \nu}=£_{\xi} g_{\mu \nu}, \quad \delta_{\xi} h_{\mu \nu}=£_{\xi} h_{\mu \nu},
$$

and a quantum gauge symmetry involving $h_{\mu \nu}$ only, that reads

$$
\begin{aligned}
\delta_{\epsilon} g_{\mu \nu} & =0, \\
\delta_{\epsilon} h_{\mu \nu} & =£_{\epsilon}\left(g_{\mu \nu}+h_{\mu \nu}\right)=\nabla_{\mu} \epsilon_{\nu}+\nabla_{\nu} \epsilon_{\mu}+\epsilon^{\lambda} \nabla_{\lambda} h_{\mu \nu}+\left(\nabla_{\mu} \epsilon^{\lambda}\right) h_{\lambda \nu}+\left(\nabla_{\nu} \epsilon^{\lambda}\right) h_{\mu \lambda} .
\end{aligned}
$$

At this point we find convenient to further split the metric fluctuation into traceless and trace parts as

$$
h_{\mu \nu} \equiv \bar{h}_{\mu \nu}+\frac{1}{D} g_{\mu \nu} h
$$

with $g^{\mu \nu} \bar{h}_{\mu \nu}=0 .^{2}$ The quadratic action in the split form reads

$S_{2}=\int d^{D} x \sqrt{g}\left\{-\frac{1}{4} \bar{h}^{\mu \nu} \nabla^{2} \bar{h}_{\mu \nu}+\left(\frac{1}{8}-\frac{1}{4 D}\right) h \nabla^{2} h-\frac{1}{2}\left[\nabla^{\nu} \bar{h}_{\nu \mu}+\left(\frac{1}{D}-\frac{1}{2}\right) \nabla_{\mu} h\right]^{2}-M_{1}-M_{2}\right\}$

\footnotetext{
${ }^{2}$ We choose to split the fields into traceless and trace pieces because the operator involved in the effective action for the full fluctuation $h_{\mu \nu}$ would produce, due to the trace projector, a non perturbative vertex in the worldline model that we prefer to avoid.
} 
where the curvature pieces $M_{1}$ and $M_{2}$ are given by

$$
\begin{aligned}
& M_{1}=\frac{1}{2} \bar{h}^{\mu \lambda} \bar{h}^{\nu \sigma} R_{\mu \nu \lambda \sigma}+\frac{1}{2}\left[\bar{h}^{\mu \lambda} \bar{h}_{\lambda}^{\nu}-\left(1-\frac{4}{D}\right) h \bar{h}^{\mu \nu}\right] R_{\mu \nu}-\frac{1}{4} \bar{h}^{\mu \nu} \bar{h}_{\mu \nu}(R-2 \Lambda), \\
& M_{2}=\left(\frac{1}{8}-\frac{3}{4 D}+\frac{1}{D^{2}}\right) h^{2} R+\left(\frac{1}{2 D}-\frac{1}{4}\right) h^{2} \Lambda .
\end{aligned}
$$

The quantum gauge symmetry for the new fields reads

$$
\delta \bar{h}_{\mu \nu}=\nabla_{\mu} \epsilon_{\nu}+\nabla_{\nu} \epsilon_{\mu}-\frac{2}{D} g_{\mu \nu} \nabla \cdot \epsilon+\mathcal{O}(h, \bar{h}), \quad \delta h=2 \nabla \cdot \epsilon+\mathcal{O}(h, \bar{h}) .
$$

We omitted the parts of the gauge transformation (2.10) linear in $h$ and $\bar{h}_{\mu \nu}$, since they are not needed at one-loop order, but are easily deduced from (2.6).

\section{Gauge fixing}

In order to deal with the action $S_{2}$ at the quantum level, we have to fix the quantum gauge symmetry (2.10). Let us denote

$$
f_{\mu} \equiv \nabla^{\nu} \bar{h}_{\nu \mu}+\left(\frac{1}{D}-\frac{1}{2}\right) \nabla_{\mu} h .
$$

We choose an $R_{\xi}$ gauge in order to remove the non laplacian part of the kinetic operator, namely the $f^{\mu} f_{\mu}$ part in (2.8). Hence, using BRST methods, we take as gauge fixing fermion the following functional

$$
\Psi=\int d^{D} x \sqrt{g} b^{\mu}\left[f_{\mu}-\frac{i}{2} \pi_{\mu}\right]
$$

where $b^{\mu}$ is the anti-ghost and $\pi_{\mu}$ the auxiliary field, such that $\delta_{B} b^{\mu}=i \eta \pi^{\mu}, \delta_{B} \pi_{\mu}=0$, $\eta$ being the anticommuting BRST parameter. The gauge fixing plus ghost actions are obtained now by the BRST variation of (3.2) with the BRST parameter stripped off

$$
S_{\pi}+S_{\mathrm{gh}}=\frac{\delta_{B} \Psi}{\delta \eta}=\int d^{D} x \sqrt{g}\left\{\left[\frac{\pi^{2}}{2}+i \pi^{\mu} f_{\mu}\right]-b^{\mu} s f_{\mu}\right\}
$$

where we denoted $s f_{\mu}=f_{\mu}(s \bar{h}, s h)$. Here $s \bar{h}_{\mu \nu}$ and $s h$ equal (2.10) with the gauge parameter $\epsilon^{\mu}$ replaced by the fermionic ghost $c^{\mu}$ (they correspond to the BRST transformations with the BRST parameter $\eta$ stripped off).

The path integral over $\pi_{\mu}, \int D \pi e^{-S_{\pi}}$, yields a contribution to the total action that reads $+\frac{1}{2} \int d^{D} x \sqrt{g} f^{2}$, canceling the corresponding term in $S_{2}$, as can be seen from (2.8). The total ghost action $S_{\text {gh }}$ consists of a quadratic piece $S_{b c}$ and higher order terms giving $b-c-h$ interactions, that are however irrelevant at the one-loop level. The total action quadratic in the quantum fields thus reads

$$
\begin{aligned}
S_{q} & =S_{\bar{h} h}+S_{b c}, \quad \text { where } \\
S_{\bar{h} h} & =\int d^{D} x \sqrt{g}\left\{-\frac{1}{4} \bar{h}^{\mu \nu} \nabla^{2} \bar{h}_{\mu \nu}+\left(\frac{1}{8}-\frac{1}{4 D}\right) h \nabla^{2} h-M_{1}-M_{2}\right\}, \\
S_{b c} & =-\int d^{D} x \sqrt{g} b^{\mu}\left[\nabla^{2} c_{\mu}+R_{\mu \nu} c^{\nu}\right] .
\end{aligned}
$$


By looking at (2.8) and (3.4), one can see that in four dimensions the action for $\bar{h}_{\mu \nu}$ and $h$ completely separates as $S_{\bar{h} h}=S_{\bar{h}}+S_{h}$, since the only mixing term $\left(1-\frac{4}{D}\right) h \bar{h}^{\mu \nu} R_{\mu \nu}$ vanishes for $D=4$. After functional integration, this leads to a complete factorization of the corresponding determinants, and for sake of simplicity we will focus from now on only in four dimensions. We set then $D=4$, where $S_{\bar{h} h}$ reads

$$
\begin{aligned}
S_{\bar{h} h} & =\int d^{4} x \sqrt{g}\left\{-\frac{1}{4} \bar{h}^{\mu \nu}\left(\nabla^{2}+2 \Lambda\right) \bar{h}_{\mu \nu}+\frac{1}{16} h\left(\nabla^{2}+2 \Lambda\right) h\right. \\
& \left.-\frac{1}{2} \bar{h}^{\mu \lambda} \bar{h}^{\nu \sigma} R_{\mu \nu \lambda \sigma}-\frac{1}{2} \bar{h}^{\mu \lambda} \bar{h}_{\lambda}^{\nu} R_{\mu \nu}-\frac{1}{4} \bar{h}^{\mu \nu} \bar{h}_{\mu \nu} R\right\} .
\end{aligned}
$$

\section{Effective action}

In order to obtain the one-loop effective action, one should integrate $e^{-S_{q}}$ in $D \bar{h} D h D b D c$. However, at this point one encounters a well-known issue in euclidean quantum gravity: the scalar piece of the action, $S_{h}$, has the wrong sign in the kinetic term, and this would make the gaussian integral badly divergent. This problem has been widely discussed in the literature, see for instance $[17,18]$. We choose to follow the one-loop prescription of Hawking and Gibbons, that suggests to Wick rotate the integration contour in $h$-space to make the integral converge. After doing so, the three actions to be integrated are

$$
\begin{aligned}
& S_{\bar{h}}=\int d^{4} x \sqrt{g}\left\{-\frac{1}{4} \bar{h}^{\mu \nu}\left(\nabla^{2}+2 \Lambda\right) \bar{h}_{\mu \nu}-\frac{1}{2} \bar{h}^{\mu \lambda} \bar{h}^{\nu \sigma} R_{\mu \nu \lambda \sigma}-\frac{1}{2} \bar{h}^{\mu \lambda} \bar{h}_{\lambda}^{\nu} R_{\mu \nu}-\frac{1}{4} \bar{h}^{\mu \nu} \bar{h}_{\mu \nu} R\right\} \\
& S_{h \text { rotated }}=-\frac{1}{16} \int d^{4} x \sqrt{g} h\left(\nabla^{2}+2 \Lambda\right) h, \\
& S_{b c}=-\int d^{4} x \sqrt{g} b^{\mu}\left[\nabla^{2} c_{\mu}+R_{\mu \nu} c^{\nu}\right] .
\end{aligned}
$$

At this juncture we can write the one-loop partition function for pure gravity by means of the following gauge fixed path integral

$$
\begin{aligned}
Z[g] & =\int D \bar{h} D h D b D c e^{-S_{q}} \\
& \propto \operatorname{Det}_{T T}^{-1 / 2}\left[K_{\mu \nu, \lambda \sigma}+M_{\mu \nu, \lambda \sigma}\right] \operatorname{Det}_{S}^{-1 / 2}\left[-\frac{1}{2}\left(\nabla^{2}+2 \Lambda\right)\right] \operatorname{Det}_{V}\left[G_{\nu}^{\mu}\right]
\end{aligned}
$$

where the subscripts of the determinants denote the functional space on which the operators are meant to act, namely symmetric traceless rank two tensors, scalars and vectors. The 
operators $K, M$ and $G$ are explicitly given by

$$
\begin{aligned}
K_{\mu \nu, \lambda \sigma} & =-\frac{1}{4}\left(g_{\mu \lambda} g_{\nu \sigma}+g_{\nu \lambda} g_{\mu \sigma}-\frac{1}{2} g_{\mu \nu} g_{\lambda \sigma}\right)\left(\nabla^{2}+2 \Lambda\right) \\
M_{\mu \nu, \lambda \sigma} & =-\frac{1}{2}\left(R_{\mu \lambda \nu \sigma}+R_{\nu \lambda \mu \sigma}-\frac{1}{2} g_{\mu \nu} R_{\lambda \sigma}-\frac{1}{2} g_{\lambda \sigma} R_{\mu \nu}+\frac{1}{8} g_{\mu \nu} g_{\lambda \sigma} R\right) \\
& -\frac{1}{4}\left(g_{\nu \sigma} R_{\mu \lambda}+g_{\mu \sigma} R_{\nu \lambda}+g_{\nu \lambda} R_{\mu \sigma}+g_{\mu \lambda} R_{\nu \sigma}-g_{\mu \nu} R_{\lambda \sigma}-g_{\lambda \sigma} R_{\mu \nu}+\frac{1}{4} g_{\mu \nu} g_{\lambda \sigma} R\right) \\
& +\frac{1}{4}\left(g_{\mu \lambda} g_{\nu \sigma}+g_{\nu \lambda} g_{\mu \sigma}-\frac{1}{2} g_{\mu \nu} g_{\lambda \sigma}\right) R \\
G_{\nu}^{\mu} & =-\frac{1}{2}\left(\delta_{\nu}^{\mu} \nabla^{2}+R_{\nu}^{\mu}\right) .
\end{aligned}
$$

The form of the above operators $K$ and $M$ may appear rather cumbersome. This is just because they have to be endomorphisms mapping traceless tensors to traceless tensors. Once they act on the correct functional space, their action greatly simplifies.

The one-loop effective action is defined as $Z[g]=e^{-\Gamma[g]}$ and thus reads

$$
\Gamma[g]=\frac{1}{2}\left\{\operatorname{Tr}_{T T} \ln \left[K_{\mu \nu, \lambda \sigma}+M_{\mu \nu, \lambda \sigma}\right]+\operatorname{Tr}_{S} \ln \left[-\frac{1}{2}\left(\nabla^{2}+2 \Lambda\right)\right]-2 \operatorname{Tr}_{V} \ln \left[G_{\nu}^{\mu}\right]\right\} .
$$

In the following section we will study a worldline model able to produce (4.4).

\section{$5 \quad$ Worldline representation}

The first step towards the worldline theory is, as usual, the Schwinger proper time representation of the logarithms. Given an operator $\hat{\mathcal{O}}$, one has

$$
\operatorname{Tr} \ln \hat{\mathcal{O}}=-\int_{0}^{\infty} \frac{d \beta}{\beta} \operatorname{Tr}\left[e^{-\beta \hat{\mathcal{O}}}\right] .
$$

The effective action for gravity is thus given by the sum of three traces, that will be reinterpreted as heat kernels of quantum mechanical hamiltonians

$$
\Gamma[g]=-\frac{1}{2} \int_{0}^{\infty} \frac{d \beta}{\beta}\left\{\operatorname{Tr}\left[e^{-\beta(\hat{K}+\hat{M})}\right]+\operatorname{Tr}\left[e^{\frac{\beta}{2}\left(\nabla^{2}+2 \Lambda\right)}\right]-2 \operatorname{Tr}\left[e^{-\beta \hat{G}}\right]\right\},
$$

where the operators $\hat{K}, \hat{M}$ and $\hat{G}$ refer to the corresponding ones in (4.3). When acting on traceless symmetric tensors and vectors, respectively, they read

$$
\begin{aligned}
(\hat{K} \phi)_{\mu \nu} & =-\frac{1}{2}\left(\nabla^{2}+2 \Lambda\right) \phi_{\mu \nu} \\
(\hat{M} \phi)_{\mu \nu} & =-\frac{1}{2}\left(R_{\mu \lambda \nu \sigma}+R_{\nu \lambda \mu \sigma}-\frac{1}{2} g_{\mu \nu} R_{\lambda \sigma}\right) \phi^{\lambda \sigma}-\frac{1}{2}\left(R_{\mu}^{\lambda} \phi_{\lambda \nu}+R_{\nu}^{\lambda} \phi_{\lambda \mu}-\frac{1}{2} g_{\mu \nu} R^{\lambda \sigma} \phi_{\lambda \sigma}\right) \\
& +\frac{1}{2} R \phi_{\mu \nu} \\
(\hat{G} v)_{\mu} & =-\frac{1}{2}\left(\nabla^{2} v_{\mu}+R_{\mu \nu} v^{\nu}\right) .
\end{aligned}
$$


Since we are going to consider $\hat{K}+\hat{M}, \hat{G}$ and $-\frac{1}{2}\left(\nabla^{2}+2 \Lambda\right)$ as quantum mechanical hamiltonians, the next task is to construct two different particle models containing symmetric traceless rank two tensors and vectors in their Hilbert spaces, where the above operators have to be realized. The scalar operator is readily recognized as the well-known hamiltonian for the bosonic particle in curved space, and needs no further discussion.

\subsection{The traceless tensor model}

Hereby we will study a worldline model able to reproduce $\hat{K}+\hat{M}$ as hamiltonian, and containing symmetric traceless rank two tensors in its Hilbert space. Spacetime coordinates and conjugate momenta $x^{\mu}(t), p_{\mu}(t)$ are the phase space bosonic variables of our model. The target space is a curved four dimensional manifold endowed with the metric $g_{\mu \nu}(x)$. To realize our Hilbert space we shall introduce worldline complex fermions that are spacetime symmetric traceless rank two tensors. A few words are now in order. We could indeed use fermionic variables as $\psi^{\mu \nu}(t)$ and $\bar{\psi}_{\mu \nu}(t)$, but this would lead to several technical issues that are cumbersome to overcome. In fact, one would have spacetime dependent $\psi, \bar{\psi}$ anticommutators. This choice would produce a lot of ordering issues in fermion bilinears that one would like to avoid. We choose then to introduce a background vielbein $e_{\mu}^{a}(x)$ and spin connection $\omega_{\mu a b}(x)$, and we introduce worldline fermions that are spacetime symmetric traceless tensors with flat indices: $\psi^{a b}(t)=\psi^{b a}(t)$ and $\bar{\psi}^{a b}(t)=\bar{\psi}^{b a}(t)$, obeying $\psi_{a}^{a}=\bar{\psi}_{a}^{a}=$ 0 , where $\bar{\psi}$ 's are conjugate momenta of $\psi$ 's. We should stress that, doing this, we are in no way quantizing gravity in the frame formulation. The quantization is performed in metric formulation, as there are no quantum fluctuations of a vielbein, nor a quantum Lorentz gauge symmetry. We are simply introducing a background vielbein, that allows to flatten indices, such as $h_{a b} \equiv e_{a}^{\mu} e_{b}^{\nu} h_{\mu \nu}$ and, whenever all operators are covariant objects, one can switch flat to curved indices for free.

Upon canonical quantization these phase space variables obey the following (anti)commutation relations

$$
\left[x^{\mu}, p_{\nu}\right]=i \delta_{\nu}^{\mu}, \quad\left\{\psi^{a b}, \bar{\psi}^{c d}\right\}=\delta^{a c} \delta^{b d}+\delta^{b c} \delta^{a d}-\frac{1}{2} \delta^{a b} \delta^{c d},
$$

where $\delta^{a b}$ is the flat metric. We realize them as operators by treating $x^{\mu}$ and $\psi^{a b}$ as graded coordinates of the wave function, while the momenta are represented as derivatives thereof ${ }^{3}$

$$
g^{1 / 4} p_{\mu} g^{-1 / 4}=-i \partial_{\mu}, \quad \bar{\psi}_{a b}=\frac{\partial}{\partial \psi^{a b}} .
$$

We stress that, as the trace-free condition is imposed covariantly ( see (5.4)), $\bar{\psi}^{a b}$ should be represented as a linear combination of $\psi$ derivatives. We represent it formally as (5.5), meaning that $\frac{\partial}{\partial \psi^{a b}} \psi^{c d}=\delta_{a}^{c} \delta_{b}^{d}+\delta_{b}^{c} \delta_{a}^{d}-\frac{1}{2} \delta_{a b} \delta^{c d}$, that is indeed the identity in trace-free space. A state in the Hilbert space is represented by a wave function depending on $x$ and

\footnotetext{
${ }^{3}$ The $g$ factors appearing here ensure hermiticity of $p_{\mu}$ when using the covariant scalar product, that involves $\sqrt{g}$, and are common when considering quantum mechanics in curved space. As for the fermions, we find it useful to consider coherent states, as defined in appendix A, so that any state $|\phi\rangle$ can be represented as a wave function $\phi(x, \psi)=(\langle x|\otimes\langle\psi|) \mid \phi\rangle$.
} 
$\psi$ variables: $|\phi\rangle \sim \phi(x, \psi)$, where as usual the $\psi$ dependence is polynomial and of finite order. We can then expand the wave function as

$$
|\phi\rangle \sim \phi(x, \psi)=\sum_{n=0}^{9} \phi_{(a b)_{1} \ldots(a b)_{n}}(x) \psi^{(a b)_{1}} \ldots \psi^{(a b)_{n}} .
$$

The sum runs from 0 to 9 in four dimensions, but in generic $D$ it would be $\frac{1}{2}(D+2)(D-1)$, the number of independent components of a rank two symmetric traceless tensor. We see that, among other fields, we have in the spectrum the rank two symmetric traceless tensor $\phi_{a b}(x) \equiv \bar{h}_{a b}(x)$ we are looking for. In the following we will project out all the unwanted additional fields so, from now on, we will always consider wave functions containing $\bar{h}_{a b}$ only: $|\bar{h}\rangle \sim \bar{h}_{a b}(x) \psi^{a b}$.

In order to construct covariant derivatives, we introduce Lorentz $S O(D)$ generators

$$
M^{a b}=-M^{b a}:=\frac{1}{2}\left[\psi^{a c}, \bar{\psi}_{c}^{b}\right]-\frac{1}{2}\left[\psi^{b c}, \bar{\psi}_{c}^{a}\right]=\psi^{a} \cdot \bar{\psi}^{b}-\psi^{b} \cdot \bar{\psi}^{a},
$$

where we denoted $\psi^{a} \cdot \bar{\psi}^{b}=\psi^{a c} \bar{\psi}_{c}^{b}$. They indeed obey the $S O(D)$ algebra

$$
\left[M^{a b}, M^{c d}\right]=\delta^{b c} M^{a d}-\delta^{a c} M^{b d}-\delta^{b d} M^{a c}+\delta^{a d} M^{b c},
$$

and are used to define the covariant derivative operator as

$$
\hat{\nabla}_{\mu}:=\partial_{\mu}+\frac{1}{2} \omega_{\mu a b} M^{a b}=\partial_{\mu}+\omega_{\mu a b} \psi^{a} \cdot \bar{\psi}^{b} .
$$

We used the hat to distinguish this quantum mechanical operator, acting on wave functions, from the ordinary covariant derivative $\nabla_{\mu}$ acting on fields. In fact one has

$$
\hat{\nabla}_{\mu} \bar{h}(x, \psi)=\left(\nabla_{\mu} \bar{h}_{a b}\right) \psi^{a b}=\left(\partial_{\mu} \bar{h}_{a b}-\omega_{\mu a}^{c} \bar{h}_{c b}-\omega_{\mu b}^{c} \bar{h}_{a c}\right) \psi^{a b} .
$$

Recalling that $p_{\mu}=-i g^{-1 / 4} \partial_{\mu} g^{1 / 4}$, the operator can be written in terms of momenta as

$$
\hat{\nabla}_{\mu}=i g^{1 / 4} \pi_{\mu} g^{-1 / 4}=i g^{1 / 4}\left(p_{\mu}-i \omega_{\mu a b} \psi^{a} \cdot \bar{\psi}^{b}\right) g^{-1 / 4},
$$

in this way defining the covariant momentum $\pi_{\mu}$. One should emphasize, however, that further applications of $\hat{\nabla}$ do not produce covariant objects. For instance, applying it twice one obtains

$$
\hat{\nabla}_{\mu} \hat{\nabla}_{\nu} \bar{h}(x, \psi)=\left(\nabla_{\mu} \nabla_{\nu} \bar{h}_{a b}+\Gamma_{\mu \nu}^{\lambda} \nabla_{\lambda} \bar{h}_{a b}\right) \psi^{a b},
$$

and so on. This is obvious by inspecting (5.9), and can be also understood from the fact that $\hat{\nabla}_{\mu}|\bar{h}\rangle$ itself does not belong to the Hilbert space. Fortunately, we need only the combination of $\hat{\nabla}$ 's that gives the laplacian, and this is a true endomorphism of the Hilbert space, sending states to states

$$
\begin{aligned}
& \hat{\nabla}^{2}:=\frac{1}{\sqrt{g}} \hat{\nabla}_{\mu} g^{\mu \nu} \sqrt{g} \hat{\nabla}_{\nu}=-g^{-1 / 4} \pi_{\mu} g^{\mu \nu} g^{1 / 2} \pi_{\nu} g^{-1 / 4}, \\
& \hat{\nabla}^{2} \bar{h}(x, \psi)=\left(\nabla^{2} \bar{h}_{a b}\right) \psi^{a b} .
\end{aligned}
$$


With these operators at hand, we can finally give the quantum mechanical representation of the operators $\hat{K}$ and $\hat{M}$ of the previous section

$$
\begin{aligned}
\hat{K} & :=-\frac{1}{2}\left(\hat{\nabla}^{2}+2 \Lambda\right) \\
& =\frac{1}{2} g^{-1 / 4} \pi_{\mu} g^{\mu \nu} g^{1 / 2} \pi_{\nu} g^{-1 / 4}-\Lambda, \\
\hat{M} & :=-\frac{1}{2} R_{a b c d} \psi^{a c} \bar{\psi}^{b d}-\frac{1}{2} R_{a b} \psi^{a} \cdot \bar{\psi}^{b}+\frac{1}{2} R,
\end{aligned}
$$

indeed, acting with $\hat{M}$ on a state one gets

$$
\hat{M} \bar{h}(x, \psi)=-\left(R_{a b c d} \bar{h}^{b d}+R_{a b} \bar{h}_{c}^{b}-\frac{1}{2} R \bar{h}_{a c}\right) \psi^{a c},
$$

that is equivalent to (5.3) if one uses the symmetry and tracelessness of $\psi^{a c}$. The quantum hamiltonian for our model is thus given by $H=\hat{K}+\hat{M}$. To construct a worldline action the only missing piece is a constraint projecting out all the unwanted fields in (5.6) with $n \neq 1$. This is easily achieved by constructing the $U(1)$ generator

$$
J=\frac{1}{4}\left[\psi^{a b}, \bar{\psi}_{a b}\right]=\mathbf{N}-\frac{9}{2},
$$

where $\mathbf{N}$ counts the number of $\psi$ 's in a state $\phi(x, \psi)$. Since $J$ commutes with $H$, it can be constrained to have a determined eigenvalue by means of a worldline $U(1)$ gauge field and a Chern-Simons coupling, as it is common in spinning particle models, see e.g. [4]. The classical action for our model, in phase space and euclidean time is finally given by

$$
\begin{aligned}
S[x, p, \psi, \bar{\psi} ; A] & =\int_{0}^{\beta} d t\left[-i p_{\mu} \dot{x}^{\mu}+\frac{1}{2} \bar{\psi}_{a b} \dot{\psi}^{a b}+\frac{1}{2} g^{\mu \nu} \pi_{\mu} \pi_{\nu}-\Lambda-\frac{1}{2} R_{a b c d} \psi^{a c} \bar{\psi}^{b d}\right. \\
& \left.-\frac{1}{2} R_{a b} \psi^{a} \cdot \bar{\psi}^{b}+\frac{1}{2} R-i A\left(\frac{1}{2} \psi^{a b} \bar{\psi}_{a b}-s\right)\right]
\end{aligned}
$$

where $A(\tau)$ is a worldine gauge field enforcing $(J-s)|\bar{h}\rangle=0$. Due to the quantum orderings appearing in (5.15), the Chern-Simons coupling ensuring $\mathbf{N}=1$ is $s=-\frac{7}{2}$ or, in arbitrary $D, s=1-\frac{(D+2)(D-1)}{4}$. The classical covariant momentum reads $\pi_{\mu}=p_{\mu}-i \omega_{\mu a b} \psi^{a} \cdot \bar{\psi}^{b}$. Integrating out momenta $p_{\mu}$, we get the classical action in configuration space

$$
\begin{aligned}
S[x, \psi, \bar{\psi} ; A] & =\int_{0}^{\beta} d t\left[\frac{1}{2} g_{\mu \nu} \dot{x}^{\mu} \dot{x}^{\nu}+\frac{1}{2} \bar{\psi}_{a b}\left(D_{t}+i A\right) \psi^{a b}-\frac{1}{2} R_{a b c d} \psi^{a c} \bar{\psi}^{b d}\right. \\
& \left.-\frac{1}{2} R_{a b} \psi^{a} \cdot \bar{\psi}^{b}+\frac{1}{2} R+i A s-\Lambda\right]
\end{aligned}
$$

where the covariant time derivative for fermions is given by $D_{t} \psi^{a b}=\dot{\psi}^{a b}+\dot{x}^{\mu}\left(\omega_{\mu}{ }^{a}{ }_{c} \psi^{c b}+\right.$ $\left.\omega_{\mu}^{b}{ }_{c} \psi^{a c}\right)$.

This is a nonlinear sigma model in curved space. It is well known that path integrals for such models need a regularization, due to ill defined products of distributions in the perturbative calculations. We shall employ dimensional regularization (DR), widely studied for classes of nonlinear sigma models analogous to the present one [3, 4, 19-22]. Once the 
regularization scheme is chosen, one has to identify the correct counterterm to be added to the classical action, to be sure that its path integral corresponds to a precise quantum hamiltonian; in the present situation to $\hat{K}+\hat{M}$. In this case, we may guess that the counterterm has two sources: one is the usual bosonic one, needed to correctly represent $\nabla^{2}$ (that is $-\frac{1}{8} R$ in DR). The other comes from ordering issues of fermions. The path integral usually produces a Weyl ordering (graded-symmetric) for fermionic polynomials. While this does not affect $\omega \psi \bar{\psi}$ terms (ordering is immaterial due to antisymmetry in $\omega_{\mu a b}$ ), it produces extra contributions of the form $\xi R$ from the potential terms like Riemann $\psi \bar{\psi}$. However, to be sure of the correctness of our computations, this educated guess has been checked by directly comparing transition amplitudes obtained with operator methods and path integrals, in close analogy with the procedure employed in [22], obtaining

$$
V_{C T}=-\frac{7}{8} R,
$$

where a contribution of $-\frac{1}{8} R$ is the DR bosonic counterterm, while $-\frac{3}{4} R$ is precisely given by Weyl ordering the potential terms in $\hat{M}$. It is useful to rescale the time as $t=\beta \tau$, in order to have $\beta$ as a parameter organizing the perturbative expansion. In doing so, we rescale the fermions as $\psi \rightarrow \frac{1}{\sqrt{\beta}} \psi, \bar{\psi} \rightarrow \frac{1}{\sqrt{\beta}} \bar{\psi}$ and the gauge field as $A \rightarrow \frac{1}{\beta} A$. We shall also add to (5.17) the counterterm (5.18), and we eventually get

$$
\begin{aligned}
S_{\text {Тт }}[x, \psi, \bar{\psi} ; A] & =\frac{1}{\beta} \int_{0}^{1} d \tau\left[\frac{1}{2} g_{\mu \nu} \dot{x}^{\mu} \dot{x}^{\nu}+\frac{1}{2} \bar{\psi}_{a b}\left(D_{\tau}+i A\right) \psi^{a b}-\frac{\beta}{2} R_{a b c d} \psi^{a c} \bar{\psi}^{b d}\right. \\
& \left.-\frac{\beta}{2} R_{a b} \psi^{a} \cdot \bar{\psi}^{b}-\beta^{2}\left(\frac{3}{8} R+\Lambda\right)\right]+i s \int_{0}^{1} d \tau A .
\end{aligned}
$$

Before starting the computation of the effective action, we present the worldline model for the ghost sector.

\subsection{The vector model}

In this subsection we will construct a quantum mechanical model having $\hat{G}$ as hamiltonian operator, and containing vector fields in its Hilbert space. The graded phase space consists, as usual, of spacetime coordinates and momenta $x^{\mu}(t), p_{\mu}(t)$, accompanied by complex worldline fermions $\lambda^{a}(t)$ and $\bar{\lambda}^{a}(t)$, where $a$ is a flat Lorentz index. This construction is similar to the usual $O(2)$ spinning particle $[4,5]$, with the difference that here the states will be vector fields with no gauge symmetry, rather than gauge invariant field strengths. The canonical (anti-)commutation relations are

$$
\left[x^{\mu}, p_{\nu}\right]=i \delta_{\nu}^{\mu}, \quad\left\{\lambda^{a}, \bar{\lambda}^{b}\right\}=\delta^{a b} .
$$

Just as before we treat $x^{\mu}$ and $\lambda^{a}$ as graded coordinates, and $p_{\mu}, \bar{\lambda}^{a}$ as derivatives thereof

$$
g^{1 / 4} p_{\mu} g^{-1 / 4}=-i \partial_{\mu}, \quad \bar{\lambda}_{a}=\frac{\partial}{\partial \lambda^{a}} .
$$

The wave function consists now of a set of antisymmetric tensors

$$
|\Psi\rangle \sim \Psi(x, \lambda)=\sum_{n=0}^{4} \Psi_{a_{1} \ldots a_{n}}(x) \lambda^{a_{1}} \ldots \lambda^{a_{n}} .
$$


The vector field we are interested in sits at the $n=1$ level. As we did in the previous case, we are going to project away all the other fields in (5.21) and so we concentrate on states of the form $|v\rangle \sim v_{a}(x) \lambda^{a}$. Lorentz generators are readily constructed as $M^{a b}:=\lambda^{a} \bar{\lambda}^{b}-\lambda^{b} \bar{\lambda}^{a}$ and the covariant derivative operator reads

$$
\hat{\nabla}_{\mu}=i g^{1 / 4} \pi_{\mu} g^{-1 / 4}=i g^{1 / 4}\left(p_{\mu}-i \omega_{\mu a b} \lambda^{a} \bar{\lambda}^{b}\right) g^{-1 / 4} .
$$

The Laplace operator is the same as before when written in terms of covariant momenta

$$
\hat{\nabla}^{2}:=-g^{-1 / 4} \pi_{\mu} g^{\mu \nu} g^{1 / 2} \pi_{\nu} g^{-1 / 4},
$$

and the full hamiltonian, the operator $\hat{G}$, is given by

$$
H \equiv \hat{G}:=\frac{1}{2} g^{-1 / 4} \pi_{\mu} g^{\mu \nu} g^{1 / 2} \pi_{\nu} g^{-1 / 4}-\frac{1}{2} R_{a b} \lambda^{a} \bar{\lambda}^{b}
$$

In order to obtain the correct worldline action, we have to project on the $n=1$ sector of the Hilbert space. The $U(1)$ generator counting the tensor rank is $J:=\frac{1}{2}\left[\lambda^{a}, \bar{\lambda}_{a}\right]=\mathbf{N}-2$ ( $\mathbf{N}-\frac{D}{2}$ in $D$ dimensions). We gauge the $J$ generator by means of a worldline gauge field $a(t)$, in order to obtain $(J-s)|v\rangle=0$ for a Chern-Simons coupling $s$. Due to the quantum orderings in $J$, the $s$ coupling enforcing $\mathbf{N}=1$ is $s=-1$, or $s=1-\frac{D}{2}$ in $D$ dimensions. With all these ingredients we can write the classical phase space action in euclidean time as

$$
S[x, p, \lambda, \bar{\lambda} ; a]=\int_{0}^{\beta} d t\left[-i p_{\mu} \dot{x}^{\mu}+\bar{\lambda}_{a} \dot{\lambda}^{a}+\frac{1}{2} g^{\mu \nu} \pi_{\mu} \pi_{\nu}-\frac{1}{2} R_{a b} \lambda^{a} \bar{\lambda}^{b}-i a\left(\lambda^{a} \bar{\lambda}_{a}-s\right)\right],
$$

where $\pi_{\mu}=p_{\mu}-i \omega_{\mu a b} \lambda^{a} \bar{\lambda}^{b}$. Integrating out $p_{\mu}$ we finally get the classical action in configuration space

$$
S[x, \lambda, \bar{\lambda} ; a]=\int_{0}^{\beta} d t\left[\frac{1}{2} g_{\mu \nu} \dot{x}^{\mu} \dot{x}^{\nu}+\bar{\lambda}_{a}\left(D_{t}+i a\right) \lambda^{a}-\frac{1}{2} R_{a b} \lambda^{a} \bar{\lambda}^{b}+i a s\right],
$$

with the covariant time derivative $D_{t} \lambda^{a}=\dot{\lambda}^{a}+\dot{x}^{\mu} \omega_{\mu}{ }_{b} \lambda^{b}$. This nonlinear sigma model has to be regularized as well. We choose again dimensional regularization, and find that the correct counterterm to be added to (5.26) is

$$
V_{C T}=-\frac{3}{8} R
$$

As in the tensor model, $-\frac{1}{8} R$ comes from the bosonic part, while $-\frac{1}{4} R$ is due to Weyl ordering of the potential $R_{a b} \lambda^{a} \bar{\lambda}^{b}$. Also here we find convenient to rescale the time as $t=\beta \tau$, as well as the fermions and the gauge field. Adding also the counterterm (5.27), we get

$S_{\mathrm{V}}[x, \lambda, \bar{\lambda} ; a]=\frac{1}{\beta} \int_{0}^{1} d \tau\left[\frac{1}{2} g_{\mu \nu} \dot{x}^{\mu} \dot{x}^{\nu}+\bar{\lambda}_{a}\left(D_{\tau}+i a\right) \lambda^{a}-\frac{\beta}{2} R_{a b} \lambda^{a} \bar{\lambda}^{b}-\frac{3}{8} \beta^{2} R\right]+i s \int_{0}^{1} d \tau a$. 


\section{One-loop effective action from worldline path integrals}

We are now ready to put all ingredients together and present the complete worldline representation of one-loop quantum gravity with cosmological constant. To test it, we use it to calculate with worldline methods the first heat kernel coefficients, also known as SeeleyDeWitt coefficients, that identify the diverging terms of the effective action (that must be renormalized away to make the effective action finite).

By looking at the representations (4.4) and (5.2) for the effective action, we see that it comes from three separate contributions: from traceless tensor modes, scalar modes, and vector ghosts

$$
\Gamma[g] \propto \Gamma_{2}+\Gamma_{0}-2 \Gamma_{1},
$$

where the subscripts refer to rank two traceless tensors, scalars and vectors, respectively. The Seeley-DeWitt coefficients $a_{n}(x)$ are identified by expanding the integrands in (5.2) in powers of $\beta$ as

$$
\Gamma[g] \propto \int_{0}^{\infty} \frac{d \beta}{\beta} \int \frac{d^{D} x \sqrt{g(x)}}{(2 \pi \beta)^{D / 2}} \sum_{n=0}^{\infty} \beta^{n} a_{n}(x),
$$

where the prefactor of $\beta^{-D / 2}$ is the leading free field behavior. We will compute separately the three contributions coming from (6.1) by quantizing on the circle the worldline actions presented in the previous section. We perform a perturbative expansion for small $\beta$, that corresponds to the ultraviolet region of the QFT, and compute the Seeley-DeWitt coefficients up to order $\beta^{2}$, that identify the divergent part of the effective action in four dimensions. The worldline actions for the tensor and the vector models have a $U(1)$ gauge symmetry that has to be fixed. It is well known, see for instance $[4,5]$, that on the circle the $U(1)$ gauge field can be fixed at most to a constant $\phi$ and one is left, after gauge fixing, with an integral over the modulus $\phi$ corresponding to the gauge invariant Wilson loop: $e^{i \phi}:=e^{i \int_{0}^{1} d \tau A(\tau)}$. Since the gauge group is abelian, the Faddeev-Popov determinant is just a constant that is absorbed in the overall normalization. After taking this into account we can write down the worldline path integral representations as

$$
\begin{aligned}
\Gamma_{2} & =\int_{0}^{\infty} \frac{d \beta}{\beta} \int_{0}^{2 \pi} \frac{d \phi}{2 \pi} \int_{P} \mathcal{D} x \int_{A} D \bar{\psi} D \psi e^{-S_{\mathrm{TT}}[x, \bar{\psi}, \psi ; \phi]}, \\
\Gamma_{1} & =\int_{0}^{\infty} \frac{d \beta}{\beta} \int_{0}^{2 \pi} \frac{d \theta}{2 \pi} \int_{P} \mathcal{D} x \int_{A} D \bar{\lambda} D \lambda e^{-S_{\mathrm{V}}[x, \bar{\lambda}, \lambda ; \theta]}, \\
\Gamma_{0} & =\int_{0}^{\infty} \frac{d \beta}{\beta} \int_{P} \mathcal{D} x e^{-S_{\mathrm{S}}[x]},
\end{aligned}
$$

where covariant integration over traceless tensor fermions is defined in appendix A. Here, $S_{\mathrm{TT}}[x, \bar{\psi}, \psi ; \phi]$ and $S_{\mathrm{V}}[x, \bar{\lambda}, \lambda ; \theta]$ are the actions (5.19) and (5.28) with the gauge fields set to constant values: $A(\tau)=\phi$ and $a(\tau)=\theta$. The subscripts $P$ and $A$ stand for periodic and antiperiodic boundary conditions, needed to take traces in the bosonic and fermionic sectors, respectively. $\mathcal{D} x \sim \prod_{\tau} \sqrt{g(x(\tau))} d^{4} x(\tau)$ denotes the generally covariant measure, while the fermionic measures are flat, since our fermions are vectors or tensors with flat 
indices. Finally, the scalar action is simply given by

$$
S_{\mathrm{S}}[x]=\frac{1}{\beta} \int_{0}^{1} d \tau\left[\frac{1}{2} g_{\mu \nu} \dot{x}^{\mu} \dot{x}^{\nu}-\beta^{2}\left(\frac{1}{8} R+\Lambda\right)\right] .
$$

This is the usual bosonic action with DR counterterm, with minimal coupling and a mass term given by $\Lambda$. Its path integral is well known and will not be computed explicitly.

\subsection{Traceless tensor path integral}

We turn now to the perturbative computation of $\Gamma_{2}$. In order to compute the periodic path integral in $x$, we split the trajectory as $x^{\mu}(\tau)=x^{\mu}+q^{\mu}(\tau)$, where $x^{\mu}$ is a fixed point in spacetime and the fluctuations vanish at the boundary: $q^{\mu}(0)=q^{\mu}(1)=0$. The $x$ path integral then splits as $\int_{P} \mathcal{D} x=\int d^{4} x \sqrt{g(x)} \int_{D} \mathcal{D} q$, where $D$ stands for Dirichlet boundary conditions. To proceed further, following [23, 24], we get rid of the metric dependent measure $\mathcal{D} q$ by exponentiating the functional product $\prod_{\tau} \sqrt{g(x(\tau))}$ with bosonic ghosts $a^{\mu}(\tau)$ and fermionic ghosts $b^{\mu}(\tau), c^{\mu}(\tau)$, such that

$$
\int_{D} \mathcal{D} q=\int_{D} D q D a D b D c e^{-S_{g h}}, \quad \text { where } \quad S_{g h}=\frac{1}{\beta} \int_{0}^{1} d \tau \frac{1}{2} g_{\mu \nu}(x)\left(a^{\mu} a^{\nu}+b^{\mu} c^{\nu}\right) .
$$

Having chosen the fixed point $x^{\mu}$, we expand all the tensor fields around it, in order to perform the perturbative expansion. From $S_{\mathrm{TT}}+S_{g h}$ we can now extract the free action

$$
S_{2}=\frac{1}{2 \beta} g_{\mu \nu} \int_{0}^{1} d \tau\left(\dot{q}^{\mu} \dot{q}^{\nu}+a^{\mu} a^{\nu}+b^{\mu} c^{\nu}\right)+\frac{1}{2 \beta} \int_{0}^{1} d \tau \bar{\psi}_{a b}\left(\partial_{\tau}+i \phi\right) \psi^{a b},
$$

where $g_{\mu \nu}=g_{\mu \nu}(x)$ is the metric at the fixed point. As usual, from $S_{2}$ we extract propagators, while the remaining part of the action $S_{i n t}$ is treated perturbatively in $\beta$. It reads

$$
\begin{aligned}
S_{i n t} & =\frac{1}{\beta} \int_{0}^{1} d \tau\left\{\frac{1}{2}\left[g_{\mu \nu}(x+q)-g_{\mu \nu}(x)\right]\left(\dot{q}^{\mu} \dot{q}^{\nu}+a^{\mu} a^{\nu}+b^{\mu} c^{\nu}\right)+\omega_{\mu a b}(x+q) \dot{q}^{\mu} \psi^{a} \cdot \bar{\psi}^{b}\right. \\
& \left.-\frac{\beta}{2} R_{a b c d}(x+q) \psi^{a c} \bar{\psi}^{b d}-\frac{\beta}{2} R_{a b}(x+q) \psi^{a} \cdot \bar{\psi}^{b}-\beta^{2} \frac{3}{8} R(x+q)\right\}
\end{aligned}
$$

where the term with $\Lambda$ and the Chern-Simons part of the action have been omitted, since they completely factorize out. We denote by $\langle\ldots\rangle$ the average computed with the free action $S_{2}$

$$
\langle F\rangle:=\frac{\int_{D} D q D a D b D c \int_{A} D \bar{\psi} D \psi F e^{-S_{2}}}{\int_{D} D q D a D b D c \int_{A} D \bar{\psi} D \psi e^{-S_{2}}} .
$$

With this notation, we can write the complete path integral as

$$
\Gamma_{2}=\int_{0}^{\infty} \frac{d \beta}{\beta} e^{\beta \Lambda} \int_{0}^{2 \pi} \frac{d \phi}{2 \pi}\left(2 \cos \frac{\phi}{2}\right)^{n} e^{-i s \phi} \int \frac{d^{D} x \sqrt{g(x)}}{(2 \pi \beta)^{D / 2}}\left\langle e^{-S_{i n t}}\right\rangle,
$$

where $n=\frac{(D+2)(D-1)}{2}$, that is $n=9$ in four dimensions, and $s=1-\frac{n}{2}$. The factor $(2 \pi \beta)^{-D / 2}$ is the usual free bosonic path integral, while $\left(2 \cos \frac{\phi}{2}\right)^{n}$ is the free path integral for $n$ antiperiodic fermions, twisted with the angle $\phi$ as in (6.5). 
By means of $S_{2}$ we obtain the following two point functions

$$
\begin{aligned}
\left\langle q^{\mu}(\tau) q^{\nu}(\sigma)\right\rangle & =-\beta g^{\mu \nu} \Delta(\tau, \sigma), \\
\left\langle a^{\mu}(\tau) a^{\nu}(\sigma)\right\rangle & =\beta g^{\mu \nu} \Delta_{g h}(\tau, \sigma), \quad\left\langle b^{\mu}(\tau) c^{\nu}(\sigma)\right\rangle=-2 \beta g^{\mu \nu} \Delta_{g h}(\tau, \sigma), \\
\left\langle\psi^{a b}(\tau) \bar{\psi}^{c d}(\sigma)\right\rangle & =\beta\left(\delta^{a c} \delta^{b d}+\delta^{b c} \delta^{a d}-\frac{1}{2} \delta^{a b} \delta^{c d}\right) \Delta_{F}(\tau-\sigma, \phi),
\end{aligned}
$$

where the inverse metric is intended at the fixed point $x^{\mu}$, and the (unregulated) propagators are given by

$$
\begin{aligned}
\Delta(\tau, \sigma) & =\sigma(\tau-1) \theta(\tau-\sigma)+\tau(\sigma-1) \theta(\sigma-\tau), \\
\Delta_{g h}(\tau, \sigma) & =\partial_{\tau}^{2} \Delta(\tau, \sigma)=\delta(\tau, \sigma), \\
\Delta_{F}(z, \phi) & =\frac{e^{-i \phi z}}{2 \cos \frac{\phi}{2}}\left[e^{i \frac{\phi}{2}} \theta(z)-e^{-i \frac{\phi}{2}} \theta(-z)\right], \quad \text { with } z=\tau-\sigma .
\end{aligned}
$$

Here $\delta(\tau, \sigma)$ is the Dirac delta acting on functions vanishing at the boundaries of the segment $[0,1]$. To give a precise meaning to ill defined products and derivatives of these distributions one has to regularize them. We choose, as anticipated, dimensional regularization to compute worldline integrals. A brief discussion of the regularization scheme and more detailed properties of the propagators (6.10) are contained in appendix B. The perturbative calculation can be performed in any coordinate system so, for sake of simplicity, we choose Riemann normal coordinates (and Fock-Schwinger gauge for the spin connection) centered at the fixed point $x^{\mu}$. Our aim is to compute the Seeley-DeWitt coefficients up to order $\beta^{2}$, hence the only terms needed in the expansion are the following (obtained, for example, by using the methods of [25])

$$
\begin{aligned}
g_{\mu \nu}(x+q) & =g_{\mu \nu}+\frac{1}{3} q^{\lambda} q^{\sigma} R_{\lambda \mu \nu \sigma}+\mathcal{O}\left(q^{3}\right)+q^{\lambda} q^{\sigma} q^{\alpha} q^{\beta}\left[\frac{1}{20} \nabla_{\lambda} \nabla_{\sigma} R_{\alpha \mu \nu \beta}+\frac{2}{45} R_{\tau \lambda \sigma \mu} R_{\alpha \beta \nu}^{\tau}\right] \\
\omega_{\mu a b}(x+q) & =\frac{1}{2} q^{\nu} R_{\nu \mu a b}+\mathcal{O}\left(q^{2}\right)+q^{\nu} q^{\lambda} q^{\sigma}\left[\frac{1}{8} \nabla_{\lambda} \nabla_{\sigma} R_{\nu \mu a b}+\frac{1}{24} R_{\nu \lambda \mu}^{\tau} R_{\sigma \tau a b}\right] \\
R_{a b c d}(x+q) & =R_{a b c d}+\mathcal{O}(q)+\frac{1}{2} q^{\mu} q^{\nu} \nabla_{\mu} \nabla_{\nu} R_{a b c d}, \\
R_{a b}(x+q) & =R_{a b}+\mathcal{O}(q)+\frac{1}{2} q^{\mu} q^{\nu} \nabla_{\mu} \nabla_{\nu} R_{a b}, \quad R(x+q)=R+\mathcal{O}(q)+\frac{1}{2} q^{\mu} q^{\nu} \nabla_{\mu} \nabla_{\nu} R
\end{aligned}
$$

where all the tensors on the right hand sides are evaluated at $x$. The terms not written explicitly are those whose path integral is trivially zero, due to the odd number of quantum fields. We can now use the above expansions in the interacting action (6.6). To get terms up to order $\beta^{2}$ it is sufficient to compute

$$
\left\langle e^{-S_{i n t}}\right\rangle=1-\left\langle S_{4}\right\rangle-\left\langle S_{6}\right\rangle+\frac{1}{2}\left\langle S_{4}^{2}\right\rangle+\mathcal{O}\left(\beta^{3}\right)
$$

where $S_{n}$ refers to the number of quantum fields, and contributes as $\left\langle S_{n}\right\rangle=\mathcal{O}\left(\beta^{n / 2-1}\right)$. 
Their explicit form is

$$
\begin{aligned}
S_{4} & =\frac{1}{6 \beta} R_{\lambda \mu \nu \sigma} \int_{0}^{1} d \tau q^{\lambda} q^{\sigma}\left(\dot{q}^{\mu} \dot{q}^{\nu}+a^{\mu} a^{\nu}+b^{\mu} c^{\nu}\right)+\frac{1}{2 \beta} R_{\mu \nu a b} \int_{0}^{1} d \tau q^{\mu} \dot{q}^{\nu} \psi_{c}^{a} \bar{\psi}^{c b} \\
& -\frac{1}{2} R_{a b c d} \int_{0}^{1} d \tau \psi^{a c} \bar{\psi}^{b d}-\frac{1}{2} R_{a b} \int_{0}^{1} d \tau \psi_{c}^{a} \bar{\psi}^{c b}-\frac{3}{8} \beta R \\
S_{6} & =\frac{1}{\beta}\left[\frac{1}{40} \nabla_{\lambda} \nabla_{\sigma} R_{\alpha \mu \nu \beta}+\frac{1}{45} R_{\tau \lambda \sigma \mu} R_{\alpha \beta \nu}^{\tau}\right] \int_{0}^{1} d \tau q^{\lambda} q^{\sigma} q^{\alpha} q^{\beta}\left(\dot{q}^{\mu} \dot{q}^{\nu}+a^{\mu} a^{\nu}+b^{\mu} c^{\nu}\right) \\
& +\frac{1}{\beta}\left[\frac{1}{8} \nabla_{\lambda} \nabla_{\sigma} R_{\mu \nu a b}+\frac{1}{24} R_{\mu \lambda \nu}^{\tau} R_{\sigma \tau a b}\right] \int_{0}^{1} d \tau q^{\lambda} q^{\sigma} q^{\mu} \dot{q}^{\nu} \psi_{c}^{a} \bar{\psi}^{c b} \\
& -\frac{1}{4} \nabla_{\mu} \nabla_{\nu} R_{a b c d} \int_{0}^{1} d \tau q^{\mu} q^{\nu} \psi^{a c} \bar{\psi}^{b d}-\frac{1}{4} \nabla_{\mu} \nabla_{\nu} R_{a b} \int_{0}^{1} d \tau q^{\mu} q^{\nu} \psi_{c}^{a} \bar{\psi}^{c b} \\
& -\frac{3}{16} \beta \nabla_{\mu} \nabla_{\nu} R \int_{0}^{1} d \tau q^{\mu} q^{\nu} .
\end{aligned}
$$

We can now compute the quantum averages needed in (6.12), by using (6.13) and the propagators given in (6.9), (6.10), and we get

$$
\begin{aligned}
\left\langle e^{\left.-S_{i n t}\right\rangle}\right. & =1+\beta R\left(\frac{1}{3}+\frac{3}{4} i \tan \frac{\phi}{2}\right) \\
& +\beta^{2}\left\{\left(\frac{1}{720}+\frac{1}{16} \cos ^{-2} \frac{\phi}{2}\right) R^{\mu \nu \lambda \sigma} R_{\mu \nu \lambda \sigma}+\left(-\frac{1}{720}-\frac{3}{16} \cos ^{-2} \frac{\phi}{2}\right) R^{\mu \nu} R_{\mu \nu}\right. \\
& \left.+\left(\frac{97}{288}-\frac{7}{32} \cos ^{-2} \frac{\phi}{2}+\frac{i}{4} \tan \frac{\phi}{2}\right) R^{2}+\left(\frac{7}{240}+\frac{i}{16} \tan \frac{\phi}{2}\right) \nabla^{2} R\right\}+\mathcal{O}\left(\beta^{3}\right) .
\end{aligned}
$$

The final step are the modular integrals in (6.8), that are easily computed by means of the residue theorem, by setting $z=e^{i \phi}$. Recalling that in four dimensions $n=9$ and $s=-\frac{7}{2}$, one gets

$$
\begin{aligned}
& I_{1}=\int_{0}^{2 \pi} \frac{d \phi}{2 \pi}\left(2 \cos \frac{\phi}{2}\right)^{9} e^{i \frac{7}{2} \phi}=9, \\
& I_{2}=\int_{0}^{2 \pi} \frac{d \phi}{2 \pi}\left(2 \cos \frac{\phi}{2}\right)^{9} e^{i \frac{7}{2} \phi} \tan \frac{\phi}{2}=7 i, \\
& I_{3}=\int_{0}^{2 \pi} \frac{d \phi}{2 \pi}\left(2 \cos \frac{\phi}{2}\right)^{9} e^{i \frac{7}{2} \phi}\left(\cos \frac{\phi}{2}\right)^{-2}=4 .
\end{aligned}
$$

By using the expansion (6.14) and the integrals above we get the final result for $\Gamma_{2}$

$$
\begin{aligned}
\Gamma_{2}=\int_{0}^{\infty} \frac{d \beta}{\beta} \int \frac{d^{4} x \sqrt{g(x)}}{(2 \pi \beta)^{2}} e^{\beta \Lambda}\left\{9-\frac{9}{4} \beta R+\beta^{2}\right. & {\left[\frac{21}{80} R^{\mu \nu \lambda \sigma} R_{\mu \nu \lambda \sigma}-\frac{61}{80} R^{\mu \nu} R_{\mu \nu}\right.} \\
& \left.\left.+\frac{13}{32} R^{2}-\frac{7}{40} \nabla^{2} R\right]+\mathcal{O}\left(\beta^{3}\right)\right\} .
\end{aligned}
$$

Let us notice that the first Seeley-DeWitt coefficient $a_{0}$ gives the propagating degrees of freedom. Indeed we obtain $a_{0}=9$ for a symmetric, rank two, traceless tensor in four 
dimensions. In order to get the two polarizations of the physical graviton we shall add the contribution of the scalar mode, and subtract the ghosts piece as dictated by (6.1). At this point, we can perform the path integral of the vector model in order to obtain $\Gamma_{1}$.

\subsection{Vector path integral}

The path integral over the circle of the action (5.28) proceeds following the same steps we presented for the tensor model. By splitting the $x$ trajectory, exponentiating the nontrivial measure in $\mathcal{D} q$, and defining averages with the free action, the vector contribution to the effective action can be cast in the form

$$
\Gamma_{1}=\int_{0}^{\infty} \frac{d \beta}{\beta} \int_{0}^{2 \pi} \frac{d \theta}{2 \pi}\left(2 \cos \frac{\theta}{2}\right)^{D} e^{-i s \theta} \int \frac{d^{D} x \sqrt{g(x)}}{(2 \pi \beta)^{D / 2}}\left\langle e^{-S_{i n t}}\right\rangle
$$

with $s=1-\frac{D}{2}$. Now fermions are spacetime vectors: $\lambda^{a}, \bar{\lambda}^{a}$, hence their contribution in the free path integral is $\left(2 \cos \frac{\theta}{2}\right)^{D}$. The free action used for the averages $\langle\ldots\rangle$ is

$$
S_{2}=\frac{1}{2 \beta} g_{\mu \nu} \int_{0}^{1} d \tau\left(\dot{q}^{\mu} \dot{q}^{\nu}+a^{\mu} a^{\nu}+b^{\mu} c^{\nu}\right)+\frac{1}{\beta} \int_{0}^{1} d \tau \bar{\lambda}_{a}\left(\partial_{\tau}+i \theta\right) \lambda^{a}
$$

while the interacting action is given by

$$
\begin{aligned}
S_{\text {int }} & =\frac{1}{\beta} \int_{0}^{1} d \tau\left\{\frac{1}{2}\left[g_{\mu \nu}(x+q)-g_{\mu \nu}(x)\right]\left(\dot{q}^{\mu} \dot{q}^{\nu}+a^{\mu} a^{\nu}+b^{\mu} c^{\nu}\right)+\omega_{\mu a b}(x+q) \dot{q}^{\mu} \lambda^{a} \bar{\lambda}^{b}\right. \\
& \left.-\frac{\beta}{2} R_{a b}(x+q) \lambda^{a} \bar{\lambda}^{b}-\beta^{2} \frac{3}{8} R(x+q)\right\} .
\end{aligned}
$$

We see that the bosonic pieces are identical. All the two point functions not involving fermions $\lambda^{a}$ and $\bar{\lambda}^{a}$ are thus exactly the same of the previous subsection. The two point function for fermions reads

$$
\left\langle\lambda^{a}(\tau) \bar{\lambda}^{b}(\sigma)\right\rangle=\delta^{a b} \Delta_{F}(\tau-\sigma, \theta),
$$

where $\Delta_{F}$ is the same as in (6.10). We shall now expand the interaction part (6.19) in Riemann normal coordinates and retain only $S_{4}$ and $S_{6}$, that read

$$
\begin{aligned}
S_{4} & =\frac{1}{6 \beta} R_{\lambda \mu \nu \sigma} \int_{0}^{1} d \tau q^{\lambda} q^{\sigma}\left(\dot{q}^{\mu} \dot{q}^{\nu}+a^{\mu} a^{\nu}+b^{\mu} c^{\nu}\right)+\frac{1}{2 \beta} R_{\mu \nu a b} \int_{0}^{1} d \tau q^{\mu} \dot{q}^{\nu} \lambda^{a} \bar{\lambda}^{b} \\
& -\frac{1}{2} R_{a b} \int_{0}^{1} d \tau \lambda^{a} \bar{\lambda}^{b}-\frac{3}{8} \beta R, \\
S_{6} & =\frac{1}{\beta}\left[\frac{1}{40} \nabla_{\lambda} \nabla_{\sigma} R_{\alpha \mu \nu \beta}+\frac{1}{45} R_{\tau \lambda \sigma \mu} R_{\alpha \beta \nu}^{\tau}\right] \int_{0}^{1} d \tau q^{\lambda} q^{\sigma} q^{\alpha} q^{\beta}\left(\dot{q}^{\mu} \dot{q}^{\nu}+a^{\mu} a^{\nu}+b^{\mu} c^{\nu}\right) \\
& +\frac{1}{\beta}\left[\frac{1}{8} \nabla_{\lambda} \nabla_{\sigma} R_{\mu \nu a b}+\frac{1}{24} R_{\mu \lambda \nu}^{\tau} R_{\sigma \tau a b}\right] \int_{0}^{1} d \tau q^{\lambda} q^{\sigma} q^{\mu} \dot{q}^{\nu} \lambda^{a} \bar{\lambda}^{b} \\
& -\frac{1}{4} \nabla_{\mu} \nabla_{\nu} R_{a b} \int_{0}^{1} d \tau q^{\mu} q^{\nu} \lambda^{a} \bar{\lambda}^{b}-\frac{3}{16} \beta \nabla_{\mu} \nabla_{\nu} R \int_{0}^{1} d \tau q^{\mu} q^{\nu} .
\end{aligned}
$$


Again, the only terms to be computed in the perturbative average are $\left\langle e^{-S_{\text {int }}}\right\rangle=1-\left\langle S_{4}\right\rangle-$ $\left\langle S_{6}\right\rangle+\frac{1}{2}\left\langle S_{4}^{2}\right\rangle$, that give

$$
\begin{aligned}
\left\langle e^{\left.-S_{i n t}\right\rangle}\right. & =1+\beta R\left(\frac{1}{3}+\frac{i}{4} \tan \frac{\phi}{2}\right) \\
& +\beta^{2}\left\{\left(\frac{1}{720}-\frac{1}{192} \cos ^{-2} \frac{\phi}{2}\right) R^{\mu \nu \lambda \sigma} R_{\mu \nu \lambda \sigma}+\left(-\frac{1}{720}+\frac{1}{32} \cos ^{-2} \frac{\phi}{2}\right) R^{\mu \nu} R_{\mu \nu}\right. \\
& \left.+\left(\frac{25}{288}-\frac{1}{32} \cos ^{-2} \frac{\phi}{2}+\frac{i}{12} \tan \frac{\phi}{2}\right) R^{2}+\left(\frac{7}{240}+\frac{i}{48} \tan \frac{\phi}{2}\right) \nabla^{2} R\right\}+\mathcal{O}\left(\beta^{3}\right) .
\end{aligned}
$$

The modular integrals are easily performed and give (in $D=4$ one has $s=-1$ )

$$
\begin{aligned}
& I_{1}=\int_{0}^{2 \pi} \frac{d \theta}{2 \pi}\left(2 \cos \frac{\theta}{2}\right)^{4} e^{i \theta}=4, \\
& I_{2}=\int_{0}^{2 \pi} \frac{d \theta}{2 \pi}\left(2 \cos \frac{\theta}{2}\right)^{4} e^{i \theta} \tan \frac{\theta}{2}=2 i, \\
& I_{3}=\int_{0}^{2 \pi} \frac{d \theta}{2 \pi}\left(2 \cos \frac{\theta}{2}\right)^{4} e^{i \theta}\left(\cos \frac{\theta}{2}\right)^{-2}=4 .
\end{aligned}
$$

Putting together (6.22) and the modular integrals in (6.17) we finally get the ghost contribution

$$
\begin{aligned}
\Gamma_{1}=\int_{0}^{\infty} \frac{d \beta}{\beta} \int \frac{d^{4} x \sqrt{g(x)}}{(2 \pi \beta)^{2}}\left\{4+\frac{5}{6} \beta R+\beta^{2}[\right. & -\frac{11}{720} R^{\mu \nu \lambda \sigma} R_{\mu \nu \lambda \sigma}+\frac{43}{360} R^{\mu \nu} R_{\mu \nu} \\
& \left.\left.+\frac{1}{18} R^{2}+\frac{3}{40} \nabla^{2} R\right]+\mathcal{O}\left(\beta^{3}\right)\right\} .
\end{aligned}
$$

\subsection{Final result}

We are finally ready to put everything together, to obtain the expansion for the gravity effective action. The scalar path integral appearing in (6.3) is well known, as already mentioned, and reads

$$
\begin{aligned}
\Gamma_{0}=\int_{0}^{\infty} \frac{d \beta}{\beta} \int \frac{d^{4} x \sqrt{g(x)}}{(2 \pi \beta)^{2}} e^{\beta \Lambda}\left\{1+\frac{1}{12} \beta R+\beta^{2}\right. & {\left[\frac{1}{720} R^{\mu \nu \lambda \sigma} R_{\mu \nu \lambda \sigma}-\frac{1}{720} R^{\mu \nu} R_{\mu \nu}\right.} \\
& \left.\left.+\frac{1}{288} R^{2}+\frac{1}{120} \nabla^{2} R\right]+\mathcal{O}\left(\beta^{3}\right)\right\} .
\end{aligned}
$$

Assembling the three contributions as dictated by (6.1): $\Gamma[g] \propto \Gamma_{2}+\Gamma_{0}-2 \Gamma_{1}$, we get the final result for the gravity effective action. Expanding in $\beta$ the $\Lambda$ exponentials in $\Gamma_{2}$ and $\Gamma_{0}$, it reads

$$
\begin{gathered}
\Gamma[g] \propto \int_{0}^{\infty} \frac{d \beta}{\beta} \int \frac{d^{4} x \sqrt{g(x)}}{(2 \pi \beta)^{2}}\left\{2+\beta\left(-\frac{23}{6} R+10 \Lambda\right)+\beta^{2}\left[\frac{53}{180} R^{\mu \nu \lambda \sigma} R_{\mu \nu \lambda \sigma}-\frac{361}{360} R^{\mu \nu} R_{\mu \nu}\right.\right. \\
\left.\left.+\frac{43}{144} R^{2}-\frac{19}{60} \nabla^{2} R+5 \Lambda^{2}-\frac{13}{6} R \Lambda\right]+\mathcal{O}\left(\beta^{3}\right)\right\} .
\end{gathered}
$$


We recognize from $a_{0}=2$ the physical polarizations of the graviton. By extracting the topological Gauss-Bonnet term $E=R_{\mu \nu \lambda \sigma}^{2}-4 R_{\mu \nu}^{2}+R^{2}$, we can rewrite the logarithmic divergent part of $\Gamma[g]$ (the $\mathcal{O}\left(\beta^{2}\right)$ Seeley-DeWitt coefficient) as

$\Gamma_{d i v}[g]=\frac{1}{8 \pi^{2}}\left(\int_{1 / M^{2}}^{\infty} \frac{d \beta}{\beta}\right) \int d^{4} x \sqrt{g(x)}\left\{\frac{53}{90} E+\frac{7}{20} R^{\mu \nu} R_{\mu \nu}+\frac{1}{120} R^{2}+10 \Lambda^{2}-\frac{13}{3} R \Lambda\right\}$,

where we dropped the total derivative $\nabla^{2} R$ and inserted the UV cut-off $\beta \sim 1 / M^{2}$ in the lower limit of the proper time integral. This is the well known result for one-loop divergencies of pure gravity $[12,13]$, and shows that our worldline model correctly reproduce them. On AdS the term in curly brackets collapses to the value of $-\frac{571}{2160} R^{2}$, which may be compared with the results of [15], as indicated in the introduction. We conclude that one may use the present worldline model with confidence to calculate more demanding one-loop amplitudes in quantum gravity.

\section{Acknowledgments}

The work of FB was supported in part by the MIUR-PRIN contract 2009-KHZKRX. FB thanks the Galileo Galilei Institute for Theoretical Physics of INFN for hospitality and support extended to him during the completion of this work.

\section{A Fermionic coherent states}

Here we review some basic formulas about fermionic coherent states, suitably modified for our traceless tensor fermions $\psi^{a b}$ and $\bar{\psi}^{a b}$.

Given the fermionic operators $\psi^{a b}$ and $\bar{\psi}^{a b}$, symmetric in $(a b)$ and traceless: $\psi_{a}^{a}=$ $\bar{\psi}_{a}^{a}=0$, they obey the oscillator algebra

$$
\left\{\psi^{a b}, \bar{\psi}^{c d}\right\}=\delta^{a c} \delta^{b d}+\delta^{b c} \delta^{a d}-\frac{2}{D} \delta^{a b} \delta^{c d},
$$

where $a, b=1, \ldots, D$ are flat Lorentz indices. We treat $\psi$ 's as creation operators and $\bar{\psi}$ 's as annihilation operators with respect to the vacuum $|0\rangle$. We define coeherent states as

$$
|\bar{\eta}\rangle:=e^{\psi^{a b} \bar{\eta}_{a b} / 2}|0\rangle, \quad\langle\xi|:=\langle 0| e^{\xi^{a b} \bar{\psi}_{a b} / 2},
$$

obeying $\bar{\psi}^{a b}|\bar{\eta}\rangle=\bar{\eta}^{a b}|\bar{\eta}\rangle$, and the analogous relation for $\langle\xi|$. They are normalized as

$$
\langle\xi \mid \bar{\eta}\rangle=e^{\xi^{a b} \bar{\eta}_{a b} / 2}
$$

Since there are $n=\frac{(D+2)(D-1)}{2}$ independent $\psi$ 's and $\bar{\psi}$ 's, to define an integral over coherent states in a Lorentz invariant way, we introduce Lorentz invariant tensors, built from $\delta_{a b}$ and $\epsilon_{a_{1} \ldots a_{D}}$ that we call $Z_{(a b)_{1} \ldots(a b)_{n}}$. They have to be symmetric and traceless in each couple $(a b)_{i}$ and antisymmetric exchanging couples, but we do not need their explicit expressions. For instance, in $D=2$ one has

$$
Z_{a b c d} \propto \epsilon_{a c} \delta_{b d}+\epsilon_{a d} \delta_{b c}+\epsilon_{b d} \delta_{a c}+\epsilon_{b c} \delta_{a d} .
$$


The integration measures are then given by

$$
d \xi:=Z_{(a b)_{1} \ldots(a b)_{n}} d \xi^{(a b)_{1}} \ldots d \xi^{(a b)_{n}}, \quad d \bar{\eta}:=Z_{(a b)_{1} \ldots(a b)_{n}} d \bar{\eta}^{(a b)_{n}} \ldots d \bar{\eta}^{(a b)_{1}}
$$

so that $d \xi d \bar{\eta}=(-)^{n} d \bar{\eta} d \xi$. The $Z$ tensors are normalized as to give

$$
\begin{aligned}
& \int d \xi d \bar{\eta} e^{-\frac{1}{2} \xi^{a b} \bar{\eta}_{a b}}=1 \\
& \int d \xi d \bar{\eta} e^{-\frac{1}{2} \xi^{a b} \bar{\eta}_{a b}}|\bar{\eta}\rangle\langle\xi|=\mathbb{1} \\
& \operatorname{Tr} A=\int d \xi d \bar{\eta} e^{-\frac{1}{2} \xi^{a b} \bar{\eta}_{a b}}\langle-\xi|A| \bar{\eta}\rangle=\int d \bar{\eta} d \xi e^{\frac{1}{2} \xi^{a b} \bar{\eta}_{a b}}\langle\xi|A| \bar{\eta}\rangle .
\end{aligned}
$$

\section{B Dimensional regularization}

In this appendix we provide some details on the regularization scheme used in the manuscript, namely dimensional regularization (DR).

The quantum fields of the traceless tensor and vector models have the following two point functions (all the formulas here are valid in four dimensions)

$$
\begin{aligned}
\left\langle q^{\mu}(\tau) q^{\nu}(\sigma)\right\rangle & =-\beta g^{\mu \nu} \Delta(\tau, \sigma), \\
\left\langle a^{\mu}(\tau) a^{\nu}(\sigma)\right\rangle & =\beta g^{\mu \nu} \Delta_{g h}(\tau, \sigma), \quad\left\langle b^{\mu}(\tau) c^{\nu}(\sigma)\right\rangle=-2 \beta g^{\mu \nu} \Delta_{g h}(\tau, \sigma), \\
\left\langle\psi^{a b}(\tau) \bar{\psi}^{c d}(\sigma)\right\rangle & =\beta\left(\delta^{a c} \delta^{b d}+\delta^{b c} \delta^{a d}-\frac{1}{2} \delta^{a b} \delta^{c d}\right) \Delta_{F}(\tau-\sigma, \phi) \\
\left\langle\lambda^{a}(\tau) \bar{\lambda}^{b}(\sigma)\right\rangle & =\beta \delta^{a b} \Delta_{F}(\tau-\sigma, \theta)
\end{aligned}
$$

that descend from the quadratic actions

$$
\begin{aligned}
S_{2}^{(\mathrm{TT})} & =\frac{1}{2 \beta} g_{\mu \nu} \int_{0}^{1} d \tau\left(\dot{q}^{\mu} \dot{q}^{\nu}+a^{\mu} a^{\nu}+b^{\mu} c^{\nu}\right)+\frac{1}{2 \beta} \int_{0}^{1} d \tau \bar{\psi}_{a b}\left(\partial_{\tau}+i \phi\right) \psi^{a b}, \\
S_{2}^{(\mathrm{V})} & =\frac{1}{2 \beta} g_{\mu \nu} \int_{0}^{1} d \tau\left(\dot{q}^{\mu} \dot{q}^{\nu}+a^{\mu} a^{\nu}+b^{\mu} c^{\nu}\right)+\frac{1}{\beta} \int_{0}^{1} d \tau \bar{\lambda}_{a}\left(\partial_{\tau}+i \theta\right) \lambda^{a} .
\end{aligned}
$$

The propagators have the continuum limit displayed in (6.10), but they come from the mode expansions

$$
\begin{aligned}
& \Delta(\tau, \sigma)=\sum_{m=1}^{\infty}\left[-\frac{2}{\pi^{2} m^{2}} \sin (\pi m \tau) \sin (\pi m \sigma)\right]=(\tau-1) \sigma \theta(\tau-\sigma)+(\sigma-1) \tau \theta(\sigma-\tau), \\
& \Delta_{g h}(\tau, \sigma)=\sum_{m=1}^{\infty} 2 \sin (\pi m \tau) \sin (\pi m \sigma)=\partial_{\tau}^{2} \Delta(\tau, \sigma)=\delta(\tau, \sigma), \\
& \Delta_{F}(\tau-\sigma, \phi)=\sum_{r \in \mathbb{Z}+1 / 2} \frac{-i}{2 \pi r+\phi} e^{2 \pi i r(\tau-\sigma)}=\frac{e^{-i \phi(\tau-\sigma)}}{2 \cos \frac{\phi}{2}}\left[e^{i \frac{\phi}{2}} \theta(\tau-\sigma)-e^{-i \frac{\phi}{2}} \theta(\sigma-\tau)\right],
\end{aligned}
$$

that are easily deduced by expanding the fields with Dirichlet boundary conditions in sine series: $\phi(\tau)=\sum_{m=1}^{\infty} \phi_{m} \sin (\pi m \tau)$, and the anti-periodic fermions in half-integer modes: 
$\Psi(\tau)=\sum_{r \in \mathbb{Z}+1 / 2} \Psi_{r} e^{2 \pi i r \tau}$. All the above distributions are meant to act on functions defined on the segment $[0,1]$, and vanishing at the boundaries for the case of bosonic fields and relative ghosts, antiperiodic for the case of fermions. With the help of (B.3) we can easily find their derivatives and equal time expressions, when well defined:

$$
\begin{aligned}
& \Delta(\tau, \sigma)=\sigma-\theta(\sigma-\tau), \quad \Delta(\tau, \sigma)=\tau-\theta(\tau-\sigma), \\
& \Delta^{\bullet}(\tau, \sigma)=1-\delta(\tau, \sigma), \\
& \Delta(\tau, \tau)=\tau(\tau-1),\left.\quad \bullet(\tau, \sigma)\right|_{\tau=\sigma}=\tau-\frac{1}{2},
\end{aligned}
$$

where dots on the left-right hand side stand for derivatives with respect to the left-right variable. By symmetric sum of the fermionic series, we also deduce:

$$
\Delta_{F}(0, \phi)=\frac{i}{2} \tan \frac{\phi}{2}
$$

while, for $\tau \neq \sigma$

$$
\Delta_{F}(\tau-\sigma, \phi) \Delta_{F}(\sigma-\tau, \phi)=-\frac{1}{4} \cos ^{-2} \frac{\phi}{2} .
$$

It is well known that products and derivatives of such distributions are ill-defined, and one needs to regularize the path integral. Discretizing the propagation time one gets time slicing regularization, while mode regularization is obtained by cutting off the series in (B.3). We choose here a different route, dimensional regularization. It consists in continuing the compact time direction with the addition of $n$ non-compact extra dimensions, such that

$$
\tau \in[0,1] \rightarrow t^{\alpha}=(\tau, \mathbf{t}) \in \mathbb{R}^{n} \times[0,1] .
$$

The quadratic action, extended in $n+1$ dimensions reads

$$
S_{2}^{(\mathrm{TT})}=\frac{1}{2 \beta} g_{\mu \nu} \int_{\mathbb{R}^{n} \times[0,1]} d^{n+1} t\left(\partial_{\alpha} q^{\mu} \partial^{\alpha} q^{\nu}+a^{\mu} a^{\nu}+b^{\mu} c^{\nu}\right)+\frac{1}{2 \beta} \int_{\mathbb{R}^{n} \times[0,1]} d^{n+1} t \bar{\psi}_{a b}\left(\gamma^{\alpha} \partial_{\alpha}+i \phi\right) \psi^{a b},
$$

and similarly for the vector model, where $\partial_{\alpha}=\frac{\partial}{\partial t^{\alpha}}$, and $\gamma^{\alpha}$ are the gamma matrices in $n+1$ dimensions. The propagators in extended space are given by

$$
\begin{aligned}
\Delta(t, s) & =\int \frac{d^{n} k}{(2 \pi)^{n}} \sum_{m=1}^{\infty} \frac{-2}{(\pi m)^{2}+\mathbf{k}^{2}} \sin (\pi m \tau) \sin (\pi m \sigma) e^{i \mathbf{k} \cdot(\mathbf{t}-\mathbf{s})}, \\
\Delta_{g h}(t, s) & =\int \frac{d^{n} k}{(2 \pi)^{n}} \sum_{m=1}^{\infty} 2 \sin (\pi m \tau) \sin (\pi m \sigma) e^{i \mathbf{k} \cdot(\mathbf{t}-\mathbf{s})} \\
& =\delta(\tau, \sigma) \delta^{n}(\mathbf{t}-\mathbf{s}), \\
\Delta_{F}(t-s, \phi) & =-i \int \frac{d^{n} k}{(2 \pi)^{n}} \sum_{r \in \mathbb{Z}+1 / 2} \frac{2 \pi r \gamma^{0}+\mathbf{k} \cdot \gamma-\phi}{(2 \pi r)^{2}+\mathbf{k}^{2}-\phi^{2}} e^{2 \pi i r(\tau-\sigma)} e^{i \mathbf{k} \cdot(\mathbf{t}-\mathbf{s})} .
\end{aligned}
$$

The propagators in extended space obey the following Green's equations

$$
\begin{array}{r}
\partial^{\alpha} \partial_{\alpha} \Delta(t, s)=\Delta_{g h}(t, s)=\delta(\tau, \sigma) \delta^{n}(\mathbf{t}-\mathbf{s}), \\
\left(\gamma^{\alpha} \frac{\partial}{\partial t^{\alpha}}+i \phi\right) \Delta_{F}(t-s, \phi)=\delta_{A}(\tau-\sigma) \delta^{n}(\mathbf{t}-\mathbf{s})
\end{array}
$$


where $\delta_{A}$ is the delta distribution acting on antiperiodic functions on $[0,1]$. Another useful identity, that can be obtained in $n+1$ dimensions, reads

$$
\left.\left[\left(\frac{\partial^{2}}{\partial t^{\alpha} \partial s_{\alpha}}+\frac{\partial^{2}}{\partial t^{\alpha} \partial t_{\alpha}}\right) \Delta(t, s)\right]\right|_{t=s}=\frac{\partial}{\partial \tau}\left[\left.\left(\frac{\partial}{\partial \tau} \Delta(t, s)\right)\right|_{t=s}\right] .
$$

In practical calculations, one does not employ the extended propagators (B.8). If the worldline diagram is ill-defined, one extends it with the extra dimensions of DR. In the extended space it is safe to integrate against the delta distributions and to integrate by parts, taking advantage of the non-compact dimensions and, using (B.9), (B.10) and integrations by parts, one can recast the integrals in a form that is not ambiguous anymore, namely with no products of delta distributions nor divergent quantities such as $\delta(\tau, \tau)$. At this point it is possible to remove the regularization, and the result is computed in the original one dimensional form. The ghost system $(a, b, c)$ ensures that every apparently divergent piece (coming from $\dot{q} \dot{q}$ propagators) is removed after doing the allowed manipulations.

Since the fermion propagator, twisted with the $U(1)$ modulus $\phi$, is exactly the same as in [4], there are no new diagrams to be regulated, and we refer to [3, 4, 21, 26, 27] for explicit examples of actual computations in DR.

\section{References}

[1] C. Schubert, "Perturbative quantum field theory in the string inspired formalism," Phys. Rept. 355 (2001) 73 [hep-th/0101036].

[2] F. Bastianelli and A. Zirotti, "Worldline formalism in a gravitational background," Nucl. Phys. B 642 (2002) 372 [hep-th/0205182].

[3] F. Bastianelli, O. Corradini and A. Zirotti, "Dimensional regularization for $\mathrm{N}=1$ supersymmetric sigma models and the worldline formalism," Phys. Rev. D 67, 104009 (2003) [hep-th/0211134].

[4] F. Bastianelli, P. Benincasa and S. Giombi, "Worldline approach to vector and antisymmetric tensor fields," JHEP 0504 (2005) 010 [hep-th/0503155].

[5] F. Bastianelli, P. Benincasa and S. Giombi, "Worldline approach to vector and antisymmetric tensor fields. II.," JHEP 0510 (2005) 114 [hep-th/0510010].

[6] F. Bastianelli and C. Schubert, "One loop photon-graviton mixing in an electromagnetic field: Part 1," JHEP 0502 (2005) 069 [gr-qc/0412095].

[7] V. D. Gershun and V. I. Tkach, "Classical and quantum dynamics of particles with arbitrary spin," JETP Lett. 29 (1979) 288 [Pisma Zh. Eksp. Teor. Fiz. 29 (1979) 320].

[8] P. S. Howe, S. Penati, M. Pernici and P. K. Townsend, "A particle mechanics description of antisymmetric tensor fields," Class. Quant. Grav. 6 (1989) 1125.

[9] S. M. Kuzenko and Z. .VYarevskaya, "Conformal invariance, N extended supersymmetry and massless spinning particles in anti-de Sitter space," Mod. Phys. Lett. A 11 (1996) 1653 [hep-th/9512115].

[10] F. Bastianelli, O. Corradini and E. Latini, "Spinning particles and higher spin fields on (A)dS backgrounds," JHEP 0811 (2008) 054 [arXiv:0810.0188 [hep-th]]. 
[11] P. Dai, Y. -t. Huang and W. Siegel, "Worldgraph approach to Yang-Mills amplitudes from N=2 spinning particle," JHEP 0810 (2008) 027 [arXiv:0807.0391 [hep-th]].

[12] G. 't Hooft and M. J. G. Veltman, "One loop divergencies in the theory of gravitation," Annales Poincare Phys. Theor. A 20 (1974) 69.

[13] S. M. Christensen and M. J. Duff, "Quantizing gravity with a cosmological constant," Nucl. Phys. B 170 (1980) 480.

[14] G. de Berredo-Peixoto, A. Penna-Firme and I. L. Shapiro, "One loop divergences of quantum gravity using conformal parametrization," Mod. Phys. Lett. A 15 (2000) 2335 [gr-qc/0103043].

[15] F. Bastianelli, R. Bonezzi, O. Corradini and E. Latini, "Effective action for higher spin fields on (A)dS backgrounds," JHEP 1212 (2012) 113 [arXiv:1210.4649 [hep-th]].

[16] M. J. Duff and P. van Nieuwenhuizen, "Quantum inequivalence of different field representations," Phys. Lett. B 94 (1980) 179.

[17] G. W. Gibbons, S. W. Hawking, M. J. Perry, "Path integrals and the indefiniteness of the gravitational action," Nucl. Phys. B 138 (1978) 141.

[18] P. O. Mazur, E. Mottola, "The gravitational measure, solution of the conformal factor problem and stability of the ground state of quantum gravity," Nucl. Phys. B 341 (1990) 187.

[19] H. Kleinert, A. Chervyakov, "Reparametrization invariance of path integrals," Phys. Lett. B 464 (1999) 257 [hep-th/9906156].

[20] F. Bastianelli, O. Corradini, P. van Nieuwenhuizen, "Dimensional regularization of the path integral in curved space on an infinite time interval," Phys. Lett. B 490 (2000) 154 [hep-th/0007105].

[21] F. Bastianelli, O. Corradini, P. van Nieuwenhuizen, "Dimensional regularization of nonlinear sigma models on a finite time interval," Phys. Lett. B 494 (2000) 161 [hep-th/0008045].

[22] F. Bastianelli, R. Bonezzi, O. Corradini, E. Latini, "Extended SUSY quantum mechanics: transition amplitudes and path integrals," JHEP 1106 (2011) 023 [arXiv:1103.3993 [hep-th]].

[23] F. Bastianelli, "The path integral for a particle in curved spaces and Weyl anomalies," Nucl. Phys. B 376 (1992) 113 [hep-th/9112035].

[24] F. Bastianelli and P. van Nieuwenhuizen, "Trace anomalies from quantum mechanics," Nucl. Phys. B 389 (1993) 53 [hep-th/9208059].

[25] U. Muller, C. Schubert, A. M. E. van de Ven, "A closed formula for the Riemann normal coordinate expansion," Gen. Rel. Grav. 31 (1999) 1759 [gr-qc/9712092].

[26] F. Bastianelli, O. Corradini, "6-D trace anomalies from quantum mechanical path integrals," Phys. Rev. D 63 (2001) 065005 [hep-th/0010118].

[27] F. Bastianelli, P. van Nieuwenhuizen, "Path integrals and anomalies in curved space," Cambridge University Press, Cambridge UK (2006). 